\title{
Detection and Measurement of magnetic data for short length wireless communication using FT-IR
}

\author{
Md. Abu Saleh \\ Department of Electronics and Telecommunication Engineering \\ Daffodil International University \\ Dhaka, Bangladesh \\ abusalehete@gmail.com,rubel_ete@diu.edu.bd
}

\begin{abstract}
Infrared (IR) radiation is a type of electromagnetic radiation. Infrared "light" has a longer wavelength than visible light. Red light has a longer wavelength than other colors of light, and infrared has even longer waves than red does; so infrared is sort of "redder-than-red" light or "beyond red" light. Infrared radiation lies between visible light and radio waves on the electromagnetic spectrum. In this paper, the infrared radiation is used for detecting the magnetic data for high speedy short range wireless communication. But infrared radiation may use in various way. This paper contains the performance of the FT-IR technique that is for multiplexing the transmissions of different users are viewed at the same time.
\end{abstract}

Keywords: FT; FT-IR; Spectrum; prism.

\section{INTRODUCTION}

The Fourier transform (often shortened FT) is an operation that transforms one function into another. In such applications as signal processing, the domain of the original function is called the time domain. In shortly FT can convert a function from one domain to another with no loss of information. And the infrared is the

$f(x)=a_{0}+\sum_{n=1}^{\infty}\left(a_{n} \cos \frac{n \pi x}{L}+b_{n} \sin \frac{n \pi x}{L}\right)$

Fig: Equation for Fourier transforms

And the infrared is the portion of the electromagnetic spectrum with wavelengths ranging from longer than visible radiation, starting around $0.7 \mu \mathrm{m}$ to $1000 \mu \mathrm{m}$ [3], to wavelengths shorter than those in the microwave. Satellite instruments typically measure infrared radiation between wavelengths of about $3 \mu \mathrm{m}$ and $20 \mu \mathrm{m}$.

The Fourier transform is also used in other kinds of spectroscopy, e.g. infrared (FT-IR). In signal is acquired in the time domain and Fourier-transformed to a Lorentzian line-shape in the frequency domain. The Fourier transform is also used in magnetic resonance imaging (MRI)[4]. In figure: 1 shows that Fourier transform (compared with Prism) can convert one signal to various signals.

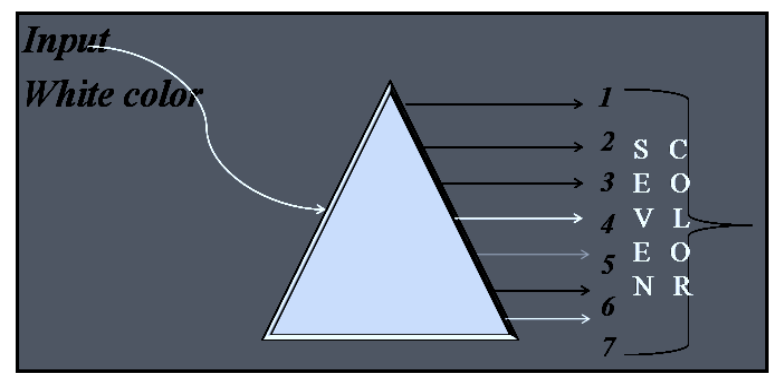

Fig. 1:- Prism, compared with Fourier Transform

An infrared spectrum represents a fingerprint [2][ii] of a sample with combination peaks which correspond to the frequencies of vibrations between the bonds of the atoms making up the material.

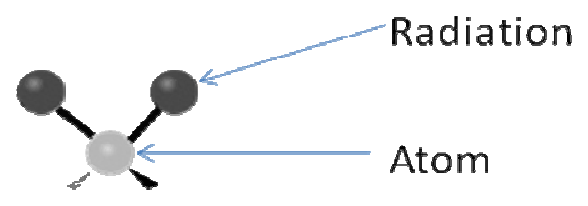

Fig. 2:- Radiate the Infrared from atom

Because each different material is a unique combination of atoms, two compounds produce the exact same infrared spectrum as shown in figure: 2 . Therefore In every material can show up a difference identity for the use of infrared spectroscopy. 


\section{CHARACTERISTICS OF FOURIER TRANSFORM INFRARED(FT-IR)}

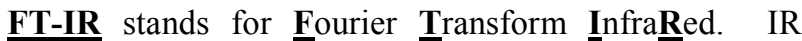
radiation is passed through a sample. Some of the infrared radiation is absorbed by the sample and some of it is passed through the transmitter...

FT-IR provides [1]:

A. It can identify unknown materials.

B. It can determine the quality or consistency of a sample.

C. It can determine the amount of components in a mixture.

D. It can increase speed, collect a scan every second

E. It is a non-negative technique

F. It provides a precise measurement method which requires no external calibration

G. It can increase sensitivity - one second scans can be co-added together to ratio out random noise.

H. It has greater optical throughput.

I. It is mechanically simple with only one moving part.

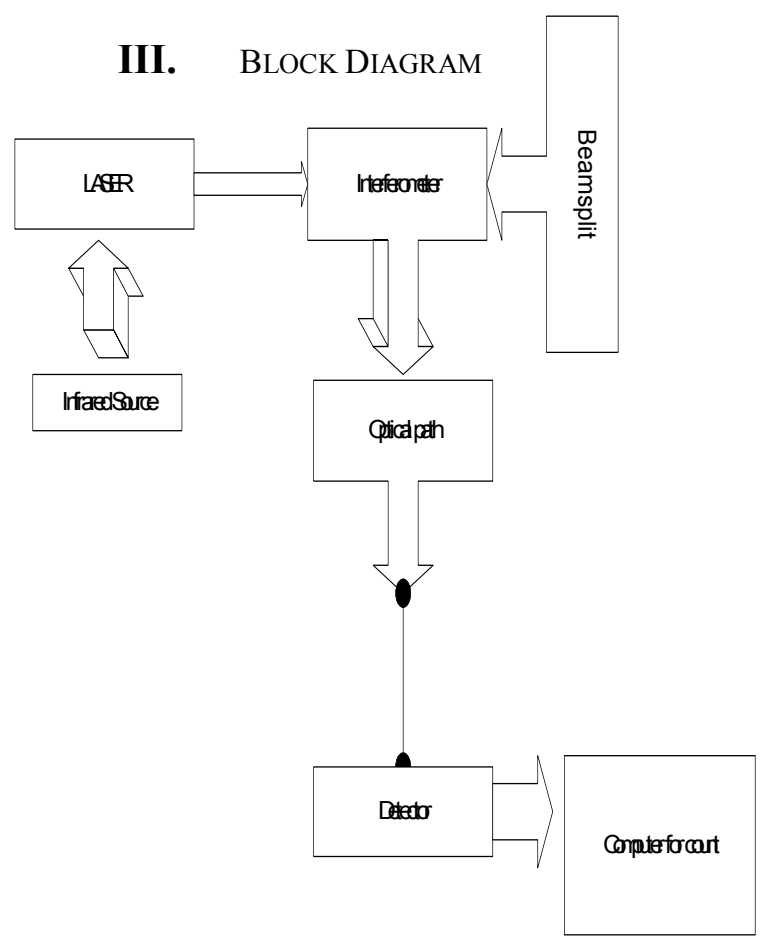

Fig. 3:- General working block diagram

\section{DESCRIPTION}

At first when the source of infrared with the combination of LASER called INFRALAS, the beam light will reflect the path through the Mirror up to detector. We should know
Vol. 1, No. 2, 2010

about the Infrared Spectroscopy. When the main door of any other way is designed with the detector sample (infralas), the INFRALAS will be capture in every second data. When some INFRALAS magnet passing through the way of door, then the detector of beam will captured the magnet and send it to the computer for collecting the data for future use. We have to send the noise detector with the optical path for reducing the noise for getting our required output to save the computer. Say, we have saved more and more data against one person. So we have to calculate the 1st and last input data. When we save those data to the computer then we can find out our attending rate in every day in any organization. So we can also find out the total rate of attendance in every one without manual received data..

\section{The Sample Analysis Process:}

The total processes for gathering the experiences on the techniques for sample handling and basic understanding using FT-IR spectrum.

A. The Source: Infrared energy is emitted from a radiant black-body source. This beam passes through a space which controls the amount of energy presented to the sample. The infrared energy is added with LASER light for strong use of data...Because of the infrared signal is a weak signal.[1]

B. The Interferometer: The beam enters the interferometer. The resulting interferogram signal then exits the interferometer.

C. The Sample: The beam enters the sample compartment where it is transmitted through or reflected off of the surface of the sample, depending on the type of analysis being expert. This is where specific frequencies of energy, which are uniquely characteristic of the sample, are absorbed.[2][i]

D. The Detector: The beam finally passes to the detector for final measurement. The detectors used are specially designed to measure the special interferogram signal.[1]

E.The Computer: The measured signal is digitized and sent to the computer where the Fourier Transformation takes place. The final infrared spectrum is then presented to the user for interpretation and any further manipulation. 


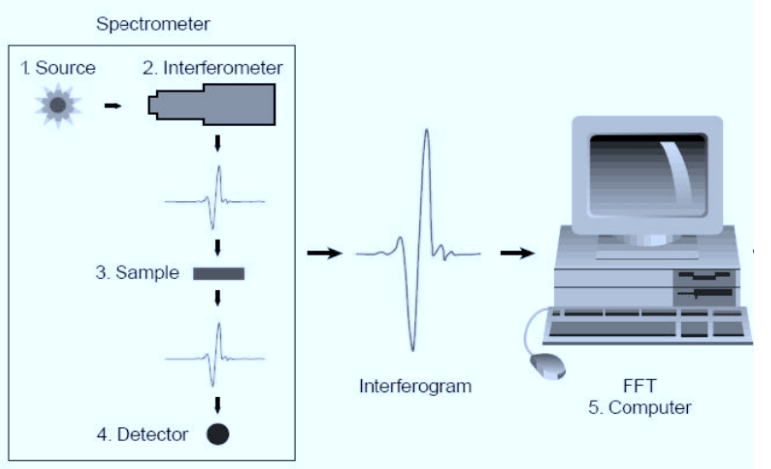

Fig. 4:- Diagram for processing control

Because there needs to be a relative scale for the absorption intensity, a background spectrum must also be measured. This is normally a measurement with no sample in the beam. This can be compared to the measurement with the sample in the beam to determine the "percent transmittance."

This technique results in a spectrum which has all of the instrumental characteristics removed.

Thus, all spectral features which are present are strictly due to the sample

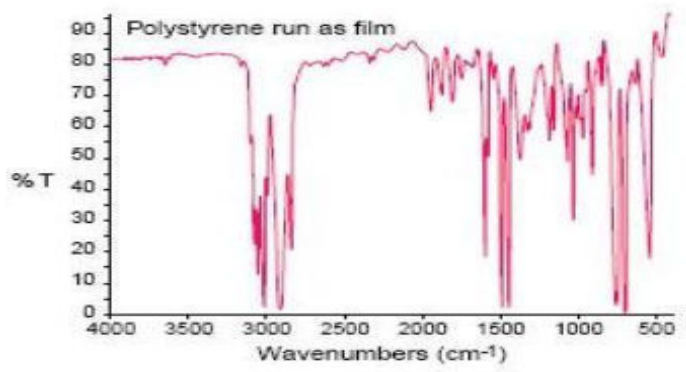

Spectrum

Fig. 5:- Wave shape for total stored data

\section{CONCLUSION}

For getting the output i.e., stored data, we have to face some problem for the sunlight. The infrared is not effective in the day light. So Infrared didn't send pure data for noise. So we have to use it in dark place i.e., indoor setup. For using indoor setup some of the major importances of FT-IR over the whole technique are speed, because all of the frequencies are measured simultaneously. The detectors employed are much more sensitive; the optical throughput is much higher which results in much lower noise levels. In FT-IR the moving mirror in the interferometer is the only continuously moving part in the instrument. Thus, there is very little possibility of mechanical breakdown. So we can say that the way to setup the infrared laser in the door detector is easy to calculate and measured the required input...

\section{REFERENCES}

[1] Thermo Nicolet, "Introduction to Fourier Transform Infrared Spectrometry”, @2001.www.thermonicolet.com

[2][i]http://www.med.harvard.edu/JPNM/physics/didactics/improc/intro/fo urier2.html

[ii] Fourier Transform Filtering Techniques,

http://www.olympusmicro.com/primer/java/digitalimaging/processing /fouriertransform/index.html

[3] Andrea, Scott and Martin, "Infrared Telescopes"

www.worldofteaching.com

http://www.jach.hawaii.edu/JACpublic/UKIRT/home.html

[4] Dictionary of the GEOS $3.9 \mu \mathrm{m}$

\section{AUTHORS PROFILE}

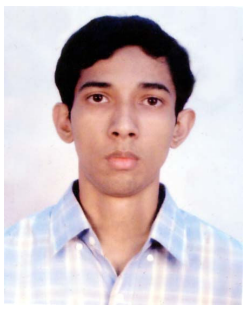

Md. Abu Saleh, a final year student of Electronics And Telecommunication Engineering at Daffodil International University, Dhaka, Bangladesh. Area of interest include Electronics, Networking, Communication, Fourier Transform, Circuit solved, Specially wireless comminication. He is a member of Bangladesh Electronics Society(BES). $\mathrm{He}$ has completed verious Electronics based project. $\mathrm{He}$ is now researching about wireless charging system. 